\title{
Does the terrestrial biosphere have planetary tipping points?
}

Barry W. Brook ${ }^{1}$, Erle C. Ellis ${ }^{2}$, Michael P. Perring ${ }^{3}$, Anson W. Mackay ${ }^{4}$, Linus Blomqvist ${ }^{5}$

${ }^{1}$ Environment Institute and School of Earth and Environmental Sciences, University of Adelaide, Adelaide, SA 5095, Australia

${ }^{2}$ Geography \& Environmental Systems, University of Maryland, Baltimore County, MD, USA

${ }^{3}$ School of Plant Biology, The University of Western Australia, Crawley, WA, Australia

${ }^{4}$ Environmental Change Research Centre, Department of Geography, UCL, London, UK

${ }^{5}$ Conservation \& Development Program, Breakthrough Institute, Oakland, CA, USA

Corresponding author: Brook, B.W. (barry.brook@adelaide.edu.au).

In Press, DOI: 10.1016/j.tree.2013.01.016

\begin{abstract}
Tipping points-where systems shift radically and potentially irreversibly into a different state-have received considerable attention in ecology. Although there is convincing evidence that human drivers can cause regime shifts at local and regional scales, the increasingly invoked concept of planetary scale tipping points in the terrestrial biosphere remains unconfirmed. By evaluating potential mechanisms and drivers, we conclude that spatial heterogeneity in drivers and responses, and lack of strong continental interconnectivity, probably induce relatively "smooth" changes at the global scale, without an expectation of marked tipping patterns. This implies that identifying critical points along global continua of drivers might be unfeasible, and that characterizing global biotic change with single aggregates is inapt.
\end{abstract}


In press (DOI 10.1016/j.tree.2013.01.016) - Embargoed

\section{Tipping points: From local to global}

The scientific concept and potential policy implications of tipping points and regime shifts have recently attracted considerable interest in global-change science [1-3]. Theoretical or empirical evidence of tipping points, manifesting on decadal to centennial time scales, exists at local and regional scales for many subsystems of the Earth system [1], including the cryosphere [4], ocean thermohaline circulation [5], atmospheric circulation [6], and marine ecosystems [7]. In the terrestrial biosphere, tipping points involve ecosystem attributes such as species abundance or carbon sequestration responding nonlinearly and potentially irreversibly to proximate drivers like habitat loss or climate change [8-12]. While discussions of ecological tipping points have heretofore been largely restricted to observations and models at local and regional scales, the recent literature has seen a number of efforts to define tipping points in terrestrial ecosystems at a global scale. This includes the framing of planetary boundaries for land-use change and biodiversity loss [13], and the suggestion that land-use change could pass a planetary tipping point that would induce a potentially irreversible global regime shift for terrestrial biodiversity [2].

Given the broad uptake of the concept of planetary tipping points, and its potential to influence research, policy, and management (see e.g. [14-17]), a rigorous examination of its application to the terrestrial biosphere-and supporting evidence-is timely. Here we put forward a definition of global-scale tipping points in the terrestrial biosphere that includes a set of testable criteria. Based on these, we then evaluate four potential drivers for such a tipping point. We conclude that the terrestrial biosphere is likely to respond heterogeneously in time and space to drivers, which implies that the pattern of change in any global aggregate ecosystem attribute will be relatively rate constant and cumulative, rather than exhibiting any identifiable tipping points at timescales shorter than geological time periods. From this, we infer that characterizing global biotic change with single global aggregates is misleading or inapt, and that identifying critical points along a continuum of forcing might not be feasible.

\section{Global ecosystem change and tipping points}

Ecosystem attributes such as species abundance or biological carbon sequestration can respond in three (stylized) ways to biotic and abiotic drivers [8-10,12]. The first type of response is characterized by being consistently proportional to the magnitude of the driver, thus exhibiting a "smooth response" pattern, where no single critical point can be determined. In the second class of ecosystem change, the response, at some critical level of forcing, is amplified by internal synergistic feedbacks and thus becomes nonlinear in relation to the driver, changing the slope of the response curve. The third class similarly involves nonlinearity, but exhibits hysteresis, in which at least two stable states exist, 
implying limited reversibility. The term "tipping point" applies to the second and third class of ecosystem change, and refers specifically to the inflection point or threshold at which the ecosystem response becomes nonlinear or the rate of change alters steeply. We define a planetary tipping point as the occurrence of this response pattern in the global aggregate of an ecosystem attribute.

Which of these three broad patterns of ecosystem change is possible at any given scale is determined principally by three characteristics: (i) the spatial homogeneity and (ii) interconnectivity of ecosystem responses, and (iii) the spatial homogeneity of drivers $[18,19]$. On the global scale, the first translates to ecosystem attributes responding similarly at the same level of pressure across continents or biomes, and the second to biotic or abiotic connections between biomes and continents, allowing localized changes to diffuse or teleconnect across the terrestrial biosphere fast enough to create a global tipping-point response (see $[20,21])$. If drivers or responses are spatially heterogeneous and inter-regional or -continental connectivity (through biotic or abiotic factors) is weak, the global aggregate pattern and rate of ecological change is likely to be relatively constant, without any identifiable tipping point. Conversely, if drivers and responses are spatially homogeneous or inter-regional or -continental connectivity is strong, ecological change might display a tipping-point pattern at global scale. One event that might fit our criteria for a global tipping point comes from the marine realm; coral reef ecosystems appear to have disappeared globally, simultaneously, and suddenly at the Triassic-Jurassic transition, likely driven by global increases in ocean acidity and temperature in response to a massive geologic carbon dioxide $\left(\mathrm{CO}_{2}\right)$ release to the atmosphere [22].

The possibility of tipping points at local to regional scales is increased by strong intra- and interspecific interactions $[23,24]$. Direct biotic interactions at these scales enable shifts in ecosystem attributes such as habitat suitability and species composition resulting from introductions or extinctions to diffuse across the entire community of organisms who interact with the species lost or gained, or whose ranges overlap with the altered habitat. However, even within well-confined ecosystems such as freshwater lakes, ecosystem responses are not necessarily homogeneous; it is even possible for only parts of freshwater lakes to shift into a turbid state [18].

There are certainly examples of direct inter-continental biotic connectivity and coupled regime shifts in terrestrial systems. For instance, intensive agriculture in western USA (habitat change) appears to have caused abrupt losses of Arctic ecosystem structure and biogeochemical cycling (biodiversity and functional loss) induced by increased populations of migrating snow geese subsidized by agricultural food supplies [25]. It is the number and strength of such connections that determine the chances of a planetary-scale tipping-point pattern. Factors that might limit direct biotic linkages include physical 
barriers to species movement, such as oceans and mountain ranges, as well as the range of climatic conditions to which specific species are adapted.

Abiotic factors that can link ecosystem change between continents include atmospheric circulation of long-lived greenhouse gases and aerosol particulates, ocean transport of heat, chemicals and sediments, and large-scale features of the hydrological cycle [26]. The climate system couples ecosystem changes at global scales over geologic time periods, including the glacial cycles [27], as do $\mathrm{CO}_{2}$ levels [28], but other strong abiotic interconnections among ecosystems have yet to be documented. Furthermore, to generate global abiotic feedbacks among ecosystems, abiotic signals must overwhelm background levels. For example, terrestrial ecosystems might experience global feedbacks through $\mathrm{CO}_{2}$ release or sequestration, if ecosystems in one region are able to significantly alter global concentrations of $\mathrm{CO}_{2}$.

We now apply these theoretical considerations to four different drivers of ecosystem change, in order to evaluate their likelihood of inducing a planetary scale tipping point.

\section{Changes in climate and atmospheric $\mathrm{CO}_{2}$}

Rising atmospheric greenhouse-gas concentrations and the climate change they cause can act as a global driver of changes in biodiversity and ecosystem processes [29], and are known to cause nonlinear responses in ecosystems on local to regional scales [30]. For instance, some global-climate models coupled to simulations of vegetation dynamics have predicted that the Amazon basin and its rainforest might exhibit a decadal-scale regime shift to drier savanna at warming of over 3-4 $\mathrm{C}^{\circ}$ [31], while boreal forests might respond non-linearly at a threshold in the 3-5 $\mathrm{C}^{\circ}$ region [1]. However, local and regional ecosystems vary considerably in their responses to climate change, and their regime shifts are therefore likely to vary considerably across the terrestrial biosphere. Apart from the direct effects of $\mathrm{CO}_{2}$ fertilization, most climatic variables that drive ecosystem responses, including temperature and precipitation, are likely to change heterogeneously across the terrestrial biosphere. Inter-continental connectivity mediated by atmospheric trace gases is similarly likely to be weak, since atmospheric $\mathrm{CO}_{2}$ changes driven by biotic changes such as vegetation shifts are far lower and slower than current rates of anthropogenic $\mathrm{CO}_{2}$ inputs from fossil fuel combustion and deforestation. Hence, the heterogeneity and independence of ecosystem responses, and the spatially variable changes in specific climatic drivers, indicate that the biospheric response would tend to be gradual, without a global tipping point at any specific level of change.

\section{Land-use change}

The conversion of terrestrial biomes into agricultural and settled landscapes has reduced the areas of habitat available to native species while altering—often negatively—many 
globally important ecological processes [32]. However, land-use change is a complex, dynamic, and physically varied process, and ecosystems response in terms of global biogeochemical cycles depend on a wide array of ecosystem characteristics, including preand post-conversion states (i.e. deforestation followed by crops, vs. regrowth), and successional dynamics. While conversion of land for human uses often reduces rates of carbon sequestration, nutrient turnover, and water cycling, it can also enhance them under some circumstances [33]. For instance, converting native grasslands to pastures by introducing livestock has little in common with forest conversion to pastures, and habitat conversions are rarely complete; most anthropogenic landscapes are heterogeneous mosaics of different land uses and land covers [34]. Indeed, these heterogeneous effects are reflected in the relatively stable aggregate levels of global net primary production (NPP) [35], and sustained or even increasing rates of carbon sequestration in the terrestrial biosphere [36] over the past half-century, even as land-use change has continued, today reaching the highest levels in human history (Figure 1) [34,37]. When assessed against our criteria (see above), the spatial unevenness and mixed aggregate impacts of humaninduced habitat loss suggest that a tipping point pattern is unlikely at the planetary scale.

\section{Habitat fragmentation}

Habitat fragmentation is known from simulations, experiments, and empirical studies to drive threshold changes in a variety of ecosystem attributes - such as population sizes and species richness of biotic communities - at local scales [38-41]. At low levels of aggregate habitat loss, when contiguous areas are reduced in area but retain connectivity, a biotic variable like abundance will typically decline proportionally with the amount of suitable habitat in a landscape. When habitats decline below a certain threshold, however, nonlinear changes emerge in response to shrinking patch size and increasing patch isolation, potentially enhanced by the Allee effect [41] and other synergies (see also $[42,43]$ ). Eventually, a tipping point is transgressed, after which patch sizes might become too small to sustain a local population, and isolation reduces dispersal ability between patches, reinforced by positive feedbacks and leading to metapopulation instability or collapse. Empirical evidence and modeling suggests that, where fragmentation thresholds exist, they tend to fall in the $10-30 \%$ range of original habitat cover $[38,41]$.

Can a threshold for habitat clearance effects on biodiversity be defined on a global scale? The fragmentation-threshold hypothesis would suggest not. First, thresholds are deeply context-dependent. The tipping point for terrestrial biotic response variables depends on landscape characteristics such as the quality of the matrix, the suitability of the patch habitat, and patch turnover rates $[41,44,45]$. Second, tipping points might differ between scales, ranging from the individual population or species, to metapopulations or aggregate species richness [41]. Finally, there is a wide range of species responses to habitat loss and 
fragmentation, depending on a species' ability to enter and utilize the matrix, its dispersal and reproductive rates, and sensitivity to habitat loss per se [41]. While populations can respond nonlinearly to habitat change, the overall richness of any given plot might not have a nonlinear (or any) response due to the heterogeneous responses of surrounding species [44]. Moreover, the loss of a particular landscape type does not necessarily result in a net reduction in species richness: one species' matrix can be another's habitat [45]. This led Lindenmayer \& Luck [44] to argue against the existence of generic rules for critical change points or threshold levels of vegetation or habitat cover across different landscapes and taxa. This conclusion is particularly valid when the focus shifts to a planetary scale. Given the variability of biotic responses, and limited biotic connectivity over larger distances, it seems unlikely that a single global threshold for a planetary regime shift can ever be determined for habitat fragmentation.

\section{Global species richness}

There is now unequivocal evidence that biodiversity loss can impair important aspects of ecosystem functioning-including resource capture, primary production, and decomposition and recycling of nutrients-as well as mounting evidence of the positive relationship between biodiversity and ecosystem stability [46]. If these relationships transpose to a planetary scale, then this would imply that global biodiversity in general, and species richness in particular, are positively correlated with the resilience and functioning of the biosphere as a whole.

However, the mechanisms behind the diversity-functioning relationship operate primarily on the local scale, at which individuals of one species interact with individuals of other species [24]. This suggests that the overall functioning of the biosphere is better described as the aggregate contribution of the many component ecosystems operating on local and regional scales, with the functional diversity and relative abundances at these scales being the biodiversity variables that influences collective global processes such as carbon sequestration, nutrient turnover, and net primary production. While global species richness in recent centuries has declined with ongoing extinction of endemics, at a regional scale, the extirpation and introduction of species occurs in parallel, sometimes resulting in net gains of species diversity, especially in naturally depauperate ecosystems like oceanic islands [47-49]. Indeed, although variable across regions, Ellis et al. [50] estimate that the net balance of introductions and extirpations is positive in about two thirds of the terrestrial biosphere. Given that both invasions and extinctions can show time lags, it is not as of yet known whether a current surplus in regional species richness will persist or be transitory [48], but there are theoretical reasons to assume that at the regional scale, species diversity will increase due to diminished influence of isolating barriers [47]. 
Consequently, on the scale at which the diversity-functioning mechanisms operate, species richness has, in many cases, increased, even as it becomes more globally homogenized, and the logical implication of the positive diversity-functioning relationship is therefore that ecosystem functioning would, on average, have benefited from this process-provided that introduced species can fulfill similar roles to the extirpated taxa [51]. This hypothesis is lent support by a meta-analysis of over 1,000 field studies, showing that while regional native species richness has often declined, primary production and several ecosystem processes were usually maintained or enhanced as a result of species introductions [52]. The extent to which this can be generalized remains unclear, because there is still substantial uncertainty as to how community and ecosystem processes respond to species introductions and losses within any one community $[49,53]$. To achieve a more mechanistic understanding of the effects of species losses and gains on ecosystem functioning, it will be necessary to better identify: i) which biological traits predispose life forms to higher probabilities of extirpation or establishment, and ii) how these response traits covary with traits that drive ecosystem functioning [46] (see also [49,54,55]). What is clear is that ecological theory does not automatically imply that a global decline in species richness will result in impaired functioning of the world's ecosystems.

We are not arguing here that extinctions do not matter, or that the health of planetary ecosystems is somehow inversely related to global biodiversity. However, we do suggest that global species richness is a poor, or even misleading indicator of Earth-system functioning and resilience. While sustaining planetary species richness is critical for conservation of evolutionary heritage and is intrinsically irreplaceable on human-relevant timescales, there is little basis in ecological theory for specifying a global aggregate number of unique species that is required to maintain the overall "health" of the biosphere.

\section{Conclusions: Effective conservation action for the biosphere in the Anthropocene}

Our examination of the evidence suggests that four principal drivers of terrestrial ecosystem change-climate change, land-use change, habitat fragmentation, and biodiversity loss-are unlikely to induce planetary scale biospheric tipping points in the terrestrial realm. Criteria that would increase the likelihood of such a global-scale tipping point-homogeneity of response over space at a short timescale, interconnectivity, and homogeneity of a causative agent across space-are not met for any of these drivers. Instead, terrestrial ecosystems are likely to respond heterogeneously to these variable forcings, and, with some few exceptions, show limited interconnectivity. If correct, our conclusion implies that ecological science is unable to define any single point along most planetary continua where the effects of global change will cause abrupt shifts or transitions to qualitatively different regimes across the whole planet. It also cautions against 
describing heterogeneous aggregates with single metrics [2,56], and highlights the problem with assuming that mechanisms and processes are necessarily transferable across local, regional and global scales.

This has two important implications for global-change science. First, we argue that focusing on uncertain future regime shifts at a planetary scale underemphasizes the degree to which ecosystems across the terrestrial biosphere have already been transformed by human activities over the long term (Box 1). Indeed, the majority of ecosystems-perhaps up to four fifths globally-have probably already undergone human-driven regime shifts of one or more kinds, at local or regional scales, over recent millennia, yielding a biosphere that today is largely characterized by post-transition, hybrid or novel ecosystems $[57,58]$ (Figure 1).

Second, framing global change in the dichotomous terms implied by the notion of a global tipping point, could lead to complacency on the "safe" side of the point, and fatalism about catastrophic or irrevocable effects on the other (see $[59,60])$. Such framing is not well supported by ecological science and can be misrepresented. (Even where tipping points have occurred on local and regional scales, there is empirical and experimental evidence to suggest that many ecosystems are able to recover even after heavy disturbance by humans $[61,62]$.) Take, for example, land-use change, where a categorization of the terrestrial biosphere into "converted" and nominally "wild" ecosystems omits human influences on non-converted land and underplays the habitat value of those landscapes already transformed by human use. As Hobbs et al. [63] suggest, "we should perhaps move away from the one-dimensional dichotomy between natural and human dominated to a more effective depiction of how human beings interact with nature." This would not only improve scientific and public understanding of the biosphere, but also foster more appropriate and pragmatic conservation strategies [64-66]. 


\section{In press (DOI 10.1016/j.tree.2013.01.016) - Embargoed}

\section{References}

1 Lenton, T. M. et al. (2008) Tipping elements in the Earth's climate system. Proc. Natl Acad. Sci U.S.A. 105, 1786-93

2 Barnosky, A. D. et al. (2012) Approaching a state shift in Earth's biosphere. Nature 486, 52-58

3 Russill, C. and Nyssa, Z. (2009) The tipping point trend in climate change communication. Global Environ. Chang. 19, 336-344

4 Winton, M. (2006) Does the Arctic sea ice have a tipping point? Geophys. Res. Lett. 33, L23504

5 Stocker, T. and Wright, D. (1991) Rapid transitions of the ocean's deep circulation induced by changes in surface water fluxes. Nature 351, 729-732

6 Claussen, M. et al. (1999) Simulation of an abrupt change in Saharan vegetation in the mid-Holocene. Geophys. Res. Lett. 26, 2037-2040

7 deYoung, B. et al. (2008) Regime shifts in marine ecosystems: detection, prediction and management. Trends Ecol. Evol. 23, 402-9

8 Andersen, T. et al. (2009) Ecological thresholds and regime shifts: approaches to identification. Trends Ecol. Evol. 24, 49-57

9 Beisner, B. et al. (2003) Alternative stable states in ecology. Front. Ecol. Environ. 1, 376-382

10 Bestelmeyer, B. T. et al. (2011) Analysis of abrupt transitions in ecological systems. Ecosphere 2, 1-26

Muradian, R. (2001) Ecological thresholds: a survey. Ecol. Econ. 38, 7-24

Scheffer, M. et al. (2001) Catastrophic shifts in ecosystems. Nature 413, 591-596

Rockström, J. et al. (2009) Planetary boundaries: exploring the safe operating space for humanity. Ecol. Soc. 14, 32

Biermann, F. (2012) Planetary boundaries and earth system governance: Exploring the links. Ecol. Econ. 81, 4-9

15 Huggett, A. J. (2005) The concept and utility of "ecological thresholds" in biodiversity conservation. Biol. Conserv. 124, 301-310

16 Lempert, R. J. and Collins, M. T. (2007) Managing the risk of uncertain threshold responses: comparison of robust, optimum, and precautionary approaches. Risk Anal. 27, 1009-1026 Polasky, S. et al. (2011) Optimal management with potential regime shifts. J. Environ. Econ. Manag. 62, 229-240 van Nes, E.H. and Scheffer, M. (2005) Implications of spatial heterogeneity for catastrophic regime shifts in ecosystems. Ecology 86, 1797-1807 Scheffer, M. et al. (2012) Anticipating Critical Transitions. Science 338, 344-348 Kinzig, A. P. P. et al. (2006) Resilience and regime shifts: assessing cascading effects. Ecol. Soc. 11, 20 Higgins, P. and Vellinga, M. (2004) Ecosystem responses to abrupt climate change: teleconnections, scale and the hydrological cycle. Climatic Change 64, 127-142 for acidification across the Triassic-Jurassic boundary. Earth-Sci. Rev. 113, 72-93 Peterson, G. et al. (1998) Ecological resilience, biodiversity, and scale. Ecosystems 1, 6-18 Tilman, D. (2000) Causes, consequences and ethics of biodiversity. Nature 405, 208-211 Jefferies, R. L. et al. (2006) A biotic agent promotes large-scale catastrophic change in the coastal marshes of Hudson Bay. J. Ecol. 94, 234-242 313-320

28 Volk, T. (2008) CO2 Rising: The World's Greatest Environmental Challenge, MIT Press.

29 Bergengren, J. C. et al. (2011) Ecological sensitivity: a biospheric view of climate change. Climatic Change 107, 433-457

30 Burkett, V. R. et al. (2005) Nonlinear dynamics in ecosystem response to climatic change: Case studies and policy implications. Ecol. Complex. 2, 357-394

31 Malhi, Y. and Aragão, L. (2009) Exploring the likelihood and mechanism of a climate-change-induced dieback of the Amazon rainforest. Proc. Natl Acad. Sci U.S.A. 106, 20610-20615

32 Foley, J. A. et al. (2005) Global consequences of land use. Science 309, 570-4

33 Guo, L. and Gifford, R. (2002) Soil carbon stocks and land use change: a meta analysis. Glob. Change Biol. 8, 345-360 


\section{In press (DOI 10.1016/j.tree.2013.01.016) - Embargoed}

Ellis, E. C. et al. (2010) Anthropogenic transformation of the biomes, 1700 to 2000. Global Ecol. Biogeogr. 19, 589-606

Running, S. W. (2012) A Measurable Planetary Boundary for the Biosphere. Science 337, 1458-1459 Pan, Y. et al. (2011) A Large and Persistent Carbon Sink in the World's Forests. Science 333, 988-993 Lepers, E. et al. (2005) A synthesis of information on rapid land-cover change for the period 19812000. BioScience 55, 115-124

Andren, H. (1994) Effects of habitat fragmentation on birds and mammals in landscapes with different proportions of suitable habitat: a review. Oikos 71,355-366

Fahrig, L. (2003) Effects of Habitat Fragmentation on Biodiversity. Annu. Rev. Ecol. Evol. S. 34, 487515

Pardini, R. et al. (2010) Beyond the fragmentation threshold hypothesis: regime shifts in biodiversity across fragmented landscapes. PLOS ONE 5, e13666

Swift, T. L. and Hannon, S. J. (2010) Critical thresholds associated with habitat loss: a review of the concepts, evidence, and applications. Biol. Rev. Camb. Philos. 85, 35-53

Brook, B. W. et al. (2008) Synergies among extinction drivers under global change. Trends Ecol. Evol. 23, 453-60

Fagan, W. F. and Holmes, E. E. (2006) Quantifying the extinction vortex. Ecol. Lett. 9, 51-60

Lindenmayer, D. B. and Luck, G. (2005) Synthesis: Thresholds in conservation and management. Biol. Conserv. 124, 351-354

Lindenmayer, D. B. and Fischer, J. (2007) Tackling the habitat fragmentation panchreston. Trends Ecol. Evol. 22, 127-132

Cardinale, B. J. et al. (2012) Biodiversity loss and its impact on humanity. Nature 486, 59-67

Sax, D. F. and Gaines, S. D. (2003) Species diversity: from global decreases to local increases. Trends Ecol. Evol. 18, 561-566

Jackson, S. T. and Sax, D. F. (2010) Balancing biodiversity in a changing environment: extinction debt, immigration credit and species turnover. Trends Ecol. Evol. 25, 153-60

Wardle, D. a et al. (2011) Terrestrial ecosystem responses to species gains and losses. Science 332, 1273-7

Ellis, E. C. et al. (2012) All Is Not Loss: Plant Biodiversity in the Anthropocene. PLoS ONE 7, e30535 Mascaro, J. et al. (2011) Novel forests maintain ecosystem processes after the decline of native tree species. Ecol. Monogr. 82, 221-228

Vilà, M. et al. (2011) Ecological impacts of invasive alien plants: a meta-analysis of their effects on species, communities and ecosystems. Ecol. Lett. 14, 702-708

Hooper, D. U. et al. (2012) A global synthesis reveals biodiversity loss as a major driver of ecosystem change. Nature 486, 105-108

Simberloff, D. et al. (2012) Impacts of biological invasions: what's what and the way forward. Trends Ecol. Evol. DOI: 10.1016/j.tree.2012.07.013

55 Pyšek, P. et al. (2012) A global assessment of invasive plant impacts on resident species, communities and ecosystems: the interaction of impact measures, invading species' traits and environment. Global Change Biol. 18, 1725-1737

Rockström, J. et al. (2009) A safe operating space for humanity. Nature 461, 472-475

Ellis, E. C. (2011) Anthropogenic transformation of the terrestrial biosphere. Philos. T. R. Soc. A. 369, 1010-1035

5 Hobbs, R. J. et al. (2009) Novel ecosystems: implications for conservation and restoration. Trends Ecol. Evol. 24, 599-605

Hulme, M. (2010) Problems with making and governing global kinds of knowledge. Global Environ. Chang. 20, 558-564

60 Nuttall, M. (2012) Tipping points and the human world: living with change and thinking about the future. Ambio 41, 96-105

Jones, H. P. and Schmitz, O. J. (2009) Rapid recovery of damaged ecosystems. PLoS ONE 4, e5653 Schröder, A. et al. (2005) Direct experimental evidence for alternative stable states: a review. Oikos. 110, 3-19

63 Hobbs, R. J. et al. (2006) Novel ecosystems: theoretical and management aspects of the new ecological world order. Global Ecol. Biogeogr. 15, 1 - 7
Sanderson, E. W. et al. (2002) The Human Foot 


\section{In press (DOI 10.1016/j.tree.2013.01.016) - Embargoed}

65 Kareiva, P. et al. (2007) Domesticated nature: shaping landscapes and ecosystems for human welfare. Science 316, 1866-1869

66 Redford, K. H. and Richter, B. D. (1999) Conservation of biodiversity in a world of use. Conserv. Biol. $13,1246-1256$

67 Brook, B. and Barnosky, A. (2012) Quaternary Extinctions and Their Link to Climate Change. In Saving a Million Species: Extinction Risk From Climate Change (Hannah, L., ed), pp. 179-198, Island Press

68 Estes, J. A et al. (2011) Trophic downgrading of planet Earth. Science 333, 301-6

69 Ellis, E. C. and Ramankutty, N. (2008) Putting people in the map: anthropogenic biomes of the world. Front. Ecol. Environ. 6, 439-447

70 Vitousek, P. M. M. et al. (1997) Introduced species: a significant component of human-caused global change. New Zeal. J. Ecol. 21, 1-16 
Figure 1. Human use of land by declining order of intensity (year 2000).

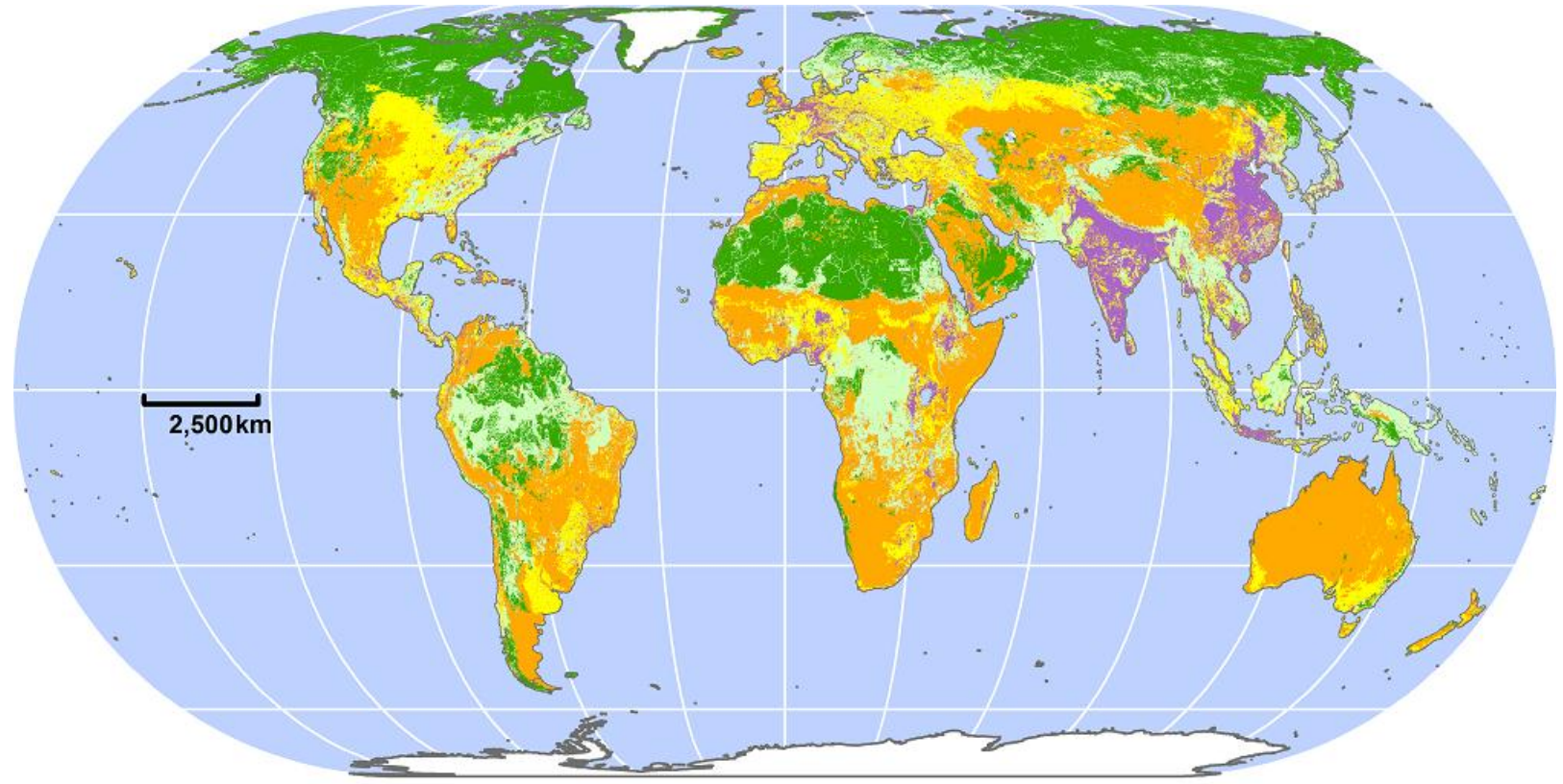

Human use of land

Dense settlements

Villages

Croplands

Rangelands

Seminatural

Wildlands

Eckert IV Projection. Data based on [34].

Detailed Legend for Human use of land

Dense settlements: Urban, suburban and other densely settled landscapes incorporate the widest variety of novel ecosystem forms and processes and are the most intensively transformed.

Villages: Densely populated agricultural landscapes, generally with ancient and intensive use of land.

Croplands: Landscapes with major use for agricultural crop production. Tending to be less completely transformed than densely settled and village landscapes, but with widespread soil tillage, agricultural inputs, domesticated species and other processes driving profound and novel ecosystem transformation.

Rangelands: Areas predominantly used for livestock grazing and forage production. Less altered than croplands, though their alteration tends to increase with population. Development of rangelands causes far more intense and novel alteration of ecosystems within woodlands than in grasslands, savannas and scrublands.

Seminatural: Landscapes with lower levels of land use and population. The least heavily used and therefore least transformed landscapes, with novel anthropogenic processes occurring primarily when population densities are highest. The most significant anthropogenic changes in these systems tend to be increased fire frequencies and the shifting of plant communities towards exotics and small numbers of domesticates.

Wildlands: Lands without evidence of human populations or use of land in global data. 


\section{Box 1: The emergence of the anthropogenic biosphere}

Humans, by the late Pleistocene, were likely a major cause of megafauna extinction across several continents [67]. Megafauna losses in the Americas, Eurasia, and Australasia altered landscapes to such an extent that the biosphere inherited by the first farmers in these regions already reflected a significant human imprint [68].

Between the Neolithic Demographic Transition (12,000 to 7,000 years ago) and the 18th Century Industrial Revolution, the human population and economy grew to make significant use of over half of the terrestrial biosphere [69]. Following the post- 1500 age of colonization, human-facilitated introductions of species into new environments worldwide also became a major cause of global change [70].

This deep interaction between people and ecosystems has resulted in a contemporary biosphere characterized by a continuum of human influence, ranging from entirely novel, human-transformed and managed ecosystems to some areas that are still largely "wild". Sanderson et al. [64] identified a "Human Footprint" on 83 \% of Earth's land surface, defined as areas influenced by one or more anthropogenic forcings such as high population density, agriculture, or infrastructure. The human impact is geographically concentrated, especially in temperate woodlands, grasslands, savannas, and shrublands (see map in Fig. 1), a majority of which have been substantially altered [57]. Indeed, a large portion of highpriority conservation areas and species, as well as a majority of global terrestrial net primary productivity, exists today in regions transformed by human use [63,69]. 
In press (DOI 10.1016/j.tree.2013.01.016) - Embargoed

\section{Glossary}

Attributes: In global-change science, attributes are measureable components of an ecosystem, either biotic or abiotic. Attributes can encapsulate features of biodiversity (e.g., species richness), community structure (e.g., species-abundance relationships), energetics (e.g., flow rates of matter), or nutrient cycling (e.g., soil carbon content).

Driver: A natural or human-caused forcing that causes change in an ecosystem. Drivers can be direct (proximate), or indirect (ultimate), depending on the perspective or scale. For example, deforestation is a proximate driver of habitat fragmentation, while economic demand for timber is an ultimate driver of deforestation, and therefore fragmentation.

Hysteresis: A situation where the current state of an ecosystem is dependent not only on its environment, but also on its history, with the return path to the original state being very different from the original development that led to the altered state. Also, at some range of the driver, there can exist two or more alternative states.

Patch and Matrix: A patch is a relatively homogenous area that differs qualitatively from its surrounding matrix, e.g., oceanic islands or forest fragments embedded in an agricultural landscape. Identification of what constitutes a patch is highly scale dependent.

Planetary Boundary: A framework concept developed by Rockström et al. [13] to define a desired operating range for essential Earth-system features and processes. Transgressing a terrestrial planetary boundary implies a risk of damaging or catastrophic loss of existing ecosystem functions or services across the entire terrestrial biosphere.

Regime Shift: A large, relatively rapid (typically annual to centennial time-scale) and persistent reorganization of the state of an ecosystem that can be triggered by synergistic feedbacks. Regime shifts that result from the crossing of tipping points are often hard to anticipate and difficult to reverse.

Smooth Response: A constant rate of change in an attribute in response to consistent changes in a driver. Contrast with a threshold response.

Synergistic Feedbacks: Some perturbations can interact to amplify the initial response. This positive feedback results in a non-linear response and can induce a tipping point. For example, habitat loss might increase the vulnerability of some species to global warming by further stressing their physiological tolerance or making range shifts impossible.

Threshold: A discontinuity whereby a small change in a driver exerts the largest change in an attribute or state of an ecosystem; this shift is typically (but not exclusively) abrupt. 
In press (DOI 10.1016/j.tree.2013.01.016) - Embargoed

Tipping Point: The critical point at which strong nonlinearities appear in the relationship between ecosystem attributes and drivers; once a tipping point threshold is crossed, the change to a new state is typically rapid and might be irreversible or exhibit hysteresis. 